\title{
Evaluation of Homolytic Dissociation Energies by Quantum Mechanical Methods
}

\author{
C. Postolache*, V. Fugaru, C. S. Tuta, G. Bubueanu \\ Horia Hulubei National Institute of Physics and Nuclear Engineering, 30, Reactorului St., Magurele, Ilfov, Romania \\ In this paper we propose a quantum mechanical evaluation model for homolytic dissociation energies. Using \\ AM1 and PM3 semi-empirical methods we have characterized molecular structures and fragments that resulted from \\ the chemical bond dissociation. Total binding energies were determined for the geometrically optimized molecular \\ structures of fragments that resulted due to the breaking of chemical bonds, as well as for transitional states. The \\ reaction enthalpy and activation energies were correlated with homolytic dissociation energies. Accuracy of the \\ method and application fields of different evaluation modalities were achieved by the intercomparison of obtained \\ values with experimental ones found in literature. More than 74 organic and inorganic compounds were analyzed.
}

DOI: 10.12693/APhysPolA.127.891

PACS: 31.15.bu

\section{Introduction}

The molecules present a significant specificity at ionizing radiation field dissociation. Experimental data suggests that chemical bonds with low values of binding energies (BE) respectively homolytic dissociation energies (HDE) are predisposed to radio-induced fragmentation $[1,2]$.

The direct or indirect experimental determination of HDE values is relatively difficult to realize and it implies time and high costs.

Specific software for quantum chemical simulation such as HYPERCHEM, CS CHEM 3D allow the construction, geometric optimization and molecular systems characterization through determination of Interatomic Distances (IAD), charge distribution, Total Binding Energies (TBE), formation enthalpies, molecular orbitals distribution, vibration analysis (IR spectrometry), electronic spectra simulation, molecular dynamics, transition states, etc.

In the presented paper, we propose an original quantum chemical model for HDE respectively BE determination by simulation of homolytic fragmentation processes.

\section{Materials and methods}

In this study, we used molecular mechanics and semiempirical computational methods. The simulation was performed on Intel Duo Core T6600 with 4 GB DD RAM computers.

Molecular structures were built and geometrically optimized using molecular mechanics methods $\mathrm{MM}+$ and AMBER (HYPERCHEM, versions 4 and 7). The energy minimizations were refined by semi-empirical methods. Depending on the chosen molecular structure the Parameterization Method 3 (PM3) [3] and Austin Method 1

\footnotetext{
* corresponding author; e-mail: cristip@nipne.ro
}

(AM1) $[4,5]$ were used. For an increase in the degree of obtained confidence, the minimizations were realized at RMS gradient values lower than $10^{-4}$. In this way it was intended to avoid local minimum points on the potential energy surface.

At first we analyzed the TBE variation for the molecular structures in relationship with the length of the analyzed chemical bonds. The IAD of analyzed bonds varied between $0.4-5 \AA$ range and TBE were computed. After that, transition state structures were obtained and thermodynamic parameters were determined. The study was performed using the Transition State computing facilities from HYPERCHEM software, versions 4 and 7 . The reaction enthalpies were determined as the difference between TBE of the geometrically optimized molecular structures and fragments resulted from chemical bonds dissociation. The activation energies were computed, using Transition States computing facility, from the difference between TBE of the reactants and reaction products.

In accordance with the obtained preliminary results, HDE can be determined from the difference between TBE of geometrically optimized molecular structures and molecular fragments after chemical bond breaking. The HDE cannot be determined from cycles using this model. In the case of molecular structures which present intra or inter molecular hydrogen bonds, the method can be applied only if the chemical bonds and fragments separation do not affect the preexistent intermolecular physical bonds.

For determining the accuracy of the proposed method, the computed values were inter-compared with experimental results identified in literature. In this sense, geometrically optimized and computed HDE were built for the following chemical compound groups: alkanes, alkenes, alkynes, halogenated derivatives, alcohols, peroxides, aldehydes, ketones, carboxylic acids, acid halogenated compounds, amines, nitro derivatives, nitrates, nitrites, thiols, and thioethers. 


\section{Results}

At first, the molecular structures of methane, ethane and benzene were geometrically optimized using PM3 and AM1 method. The $\mathrm{C}-\mathrm{C}$ (in the case of ethane) and $\mathrm{C}-\mathrm{H}$ (in the case of methane and benzene) IAD was varied in $0.4-10 \AA$ range. TBE variations were calculated using PM3 and AM1 methods (with setting options: multiplicity $=1$, spin pairing $\mathrm{UHF}$ ) revealing the presence of a minimum for the equilibrium distance, obtained by geometrical optimization. For IAD values higher than $3.5 \AA$, TBE reach a plateau. Computed values for singlet states become equal with those calculated for triplet states suggesting chemical bond breaking with the formation of two free radicals.

The computed HDE and experimentally determined BE (Exp. BE) values [6] for hydrocarbon compounds.

TABLE I

\begin{tabular}{|c|c|c|c|c|c|c|c|c|c|c|c|c|c|}
\hline \multirow{3}{*}{$\begin{array}{l}\text { Chemical compound } \\
\text { /Chemical bond } \\
\text { emi-empirical method }\end{array}$} & \multirow{2}{*}{\multicolumn{2}{|c|}{$\begin{array}{c}\text { HDE } \\
\text { unoptimized } \\
{[\mathrm{kcal} / \mathrm{mol}]}\end{array}$}} & \multirow{2}{*}{\multicolumn{2}{|c|}{$\begin{array}{c}\text { HDE } \\
\text { optimized } \\
{[\mathrm{kcal} / \mathrm{mol}]} \\
\end{array}$}} & \multicolumn{4}{|c|}{\begin{tabular}{|c|} 
HDE \\
unoptimized deviation \\
\end{tabular}} & \multicolumn{4}{|c|}{$\begin{array}{c}\text { HDE } \\
\text { optimized deviation }\end{array}$} & \multirow{3}{*}{$\begin{array}{c}\text { Exp. } \\
\text { BE } \\
{[6]}\end{array}$} \\
\hline & & & & & \multicolumn{2}{|c|}{$[\mathrm{kcal} / \mathrm{mol}]$} & \multicolumn{2}{|c|}{$\%$} & \multicolumn{2}{|c|}{$[\mathrm{kcal} / \mathrm{mol}]$} & \multicolumn{2}{|c|}{$\%$} & \\
\hline & PM3 & AM1 & PM3 & AM1 & PM3 & AM1 & PM3 & AM1 & PM3 & AM1 & PM3 & AM1 & \\
\hline Methane $/ \mathrm{H}_{3} \mathrm{C}-\mathrm{H}$ & 102.7 & 102.3 & 3.1 & 4 & 0.7 & 0 . & 0.7 & 0.3 & -8.9 & -8.6 & -8.7 & -8.4 & 102 \\
\hline Etha & 91.7 & 91.7 & 74.1 & 74.4 & 6.7 & 6.7 & 7.9 & 7.9 & -10.9 & -10.6 & -12.8 & -12.5 & 85 \\
\hline Ethan & 5.6 & 9 & 84.8 & 84.7 & -0.4 & -0.7 & -0.4 & -0.7 & -11.2 & -11.3 & -11.7 & -11.8 & 96 \\
\hline Propan & 96.3 & 96.3 & 86.1 & 86.4 & 1.3 & 1.3 & 1.4 & 1.4 & -8.9 & -8.6 & -9.4 & -9.1 & 95 \\
\hline$(\mathrm{H})\left(\mathrm{CH}_{3}\right) \mathrm{C}$ & 91.1 & 1.2 & 87.6 & 80.9 & -2.9 & -2.8 & -3.1 & -3.0 & -6.4 & -13.1 & -6.8 & -13.9 & 94 \\
\hline Propane/ $\mathrm{H}_{3} \mathrm{C}-\mathrm{CH}_{2}-\mathrm{CH}_{3}$ & 91 & 90.8 & 76.4 & 74.3 & 6 & 5.8 & 7.1 & 6.8 & -8.6 & -10.7 & -10.1 & -12.6 & 85 \\
\hline n-butane/CH & 83.5 & 84.1 & 71.3 & 68.2 & 5.5 & 6.1 & 7.1 & 7.8 & -6.7 & -9.8 & -8.6 & -12.6 & 78 \\
\hline n-butane $/ \mathrm{C}_{3} \mathrm{H}_{7}$ & 96 & 96 & 84.9 & 84.9 & 2 & 2 & 2.1 & 2.1 & -9.1 & -9.1 & -9.7 & -9.7 & 94 \\
\hline Tert-b & 88.3 & 88 & 76.5 & 72.3 & -0.7 & -1 & -0.8 & -1.1 & -12.5 & -16.7 & -14.0 & -18.8 & 89 \\
\hline Ethene/ $\mathrm{H}_{2} \mathrm{C}=(\mathrm{H}) \mathrm{C}-\mathrm{H}$ & 101.1 & 101.3 & 93.3 & 93.3 & 5.1 & 5.3 & 5.3 & 5.5 & -2.7 & -2.7 & -2.8 & -2.8 & 96 \\
\hline Acet & 128.1 & 129.1 & 33.4 & 110.9 & 7.1 & 8.1 & 5.9 & 6.7 & 12.4 & -10.1 & 10.2 & -8.3 & 121 \\
\hline Acet & 226.2 & 222.5 & 215.3 & 210.5 & -3.8 & -7.5 & -1.7 & -3.3 & -14.7 & -19.5 & -6.4 & -8.5 & 230 \\
\hline Ethylbenze & 79.8 & 81.4 & 79.2 & 85.8 & 5.8 & 7.4 & 7.8 & 10.0 & 5.2 & 11.8 & 7.0 & 15.9 & 74 \\
\hline Toluene/(Ph)C-CH 3 & 92.5 & 93.8 & 77.6 & 77.8 & 5.5 & 6.8 & 6.3 & 7.8 & -9.4 & -9.2 & -10.8 & -10.6 & 87 \\
\hline Benzene/C-H & 103.7 & 103.6 & 99.8 & 99.7 & 1.7 & 1.6 & 1.7 & 1.6 & -2.2 & -2.3 & -2.2 & -2.3 & 102 \\
\hline
\end{tabular}

The computed HDE and experimentally determined BE values [6] for allyl and benzyl positions.

TABLE II

\begin{tabular}{|c|c|c|c|c|c|c|c|c|c|c|c|c|c|}
\hline \multirow[t]{2}{*}{$\begin{array}{l}\text { Chemical compound } \\
\text { /Chemical bond }\end{array}$} & \multirow{2}{*}{\multicolumn{2}{|c|}{$\begin{array}{c}\text { HDE } \\
\text { unoptimized } \\
{[\mathrm{kcal} / \mathrm{mol}]}\end{array}$}} & \multirow{2}{*}{\multicolumn{2}{|c|}{$\begin{array}{c}\text { HDE } \\
\text { optimized } \\
{[\mathrm{kcal} / \mathrm{mol}]} \\
\end{array}$}} & \multicolumn{4}{|c|}{\begin{tabular}{|c|} 
HDE \\
unoptimized deviation
\end{tabular}} & \multicolumn{4}{|c|}{$\begin{array}{c}\text { HDE } \\
\text { optimized deviation }\end{array}$} & \multirow{3}{*}{$\begin{array}{c}\text { Exp. } \\
\text { BE } \\
{[6]}\end{array}$} \\
\hline & & & & & {$[\mathrm{kca}]$} & $/ \mathrm{mol}]$ & & & [kcal & $/ \mathrm{mol}]$ & $\%$ & & \\
\hline Semi-e & PM3 & AM1 & PM3 & AM1 & PM3 & AM1 & PM3 & AM1 & PM3 & AM1 & PM3 & AM1 & \\
\hline Toluene & 8.1 & 9 & 78.4 & 78.4 & 20.6 & 20.8 & 26.6 & 26.9 & 9 & 0.9 & 1.1 & 1.2 & 77.5 \\
\hline $\mathrm{p}-\mathrm{xyl}$ & .3 & 93.1 & 7.2 & 7.3 & 7.3 & 17.1 & 22.7 & 2.5 & 1.2 & 1.3 & 1.6 & .7 & 76 \\
\hline$-\mathrm{H}_{2} \mathrm{C}-\mathrm{CH}_{3}$ & 92.3 & 92.3 & 57.0 & 56.9 & 29.1 & 29.1 & 46.0 & 46.1 & -6.2 & -6.3 & -9.8 & -9.9 & 63.2 \\
\hline & 7.2 & 87.2 & 8.9 & 58.9 & 2.2 & 22.2 & 34.1 & 34.2 & -6.1 & -6.1 & -9.4 & -9 & 65 \\
\hline 1,2 biphe & 87.6 & 87.5 & 57.0 & 56.8 & 40.6 & 40.5 & 86.4 & 86.2 & 10.0 & 9.8 & 21.2 & 20.8 & 47 \\
\hline & 4.3 & 94.3 & 78.8 & 78.7 & 9.3 & 19.3 & 25.7 & 25.8 & 3.8 & 3.7 & 5.0 & 4.9 & 75 \\
\hline${ }_{2} \mathrm{C}-\mathrm{H}$ & 97.5 & 97.4 & 78.1 & 78.1 & 21.5 & 21.4 & 28.2 & 28.1 & 2.1 & 2.1 & 2.7 & 2.8 & 76 \\
\hline${ }_{2} \mathrm{C}-\mathrm{H}$ & 4.3 & 94.4 & 78.9 & 78.8 & 7.3 & 17.4 & 22.5 & 22.6 & 1.9 & 1.8 & 2.4 & 2.3 & 77 \\
\hline$-\mathrm{NH}_{2}$ & 72.4 & 72.3 & 54.9 & 55.0 & 13.4 & 13.3 & 22.7 & 22.6 & -4.1 & -4.0 & -7.0 & -6.8 & 59 \\
\hline & 9.0 & 68.9 & 52.2 & 52.2 & 16.0 & 15.9 & 30.2 & 30.0 & -0.8 & -0.8 & -1.6 & -1.4 & 53 \\
\hline$-\mathrm{H}_{2} \mathrm{C}-\mathrm{SC}$ & 64.3 & 64.4 & 47.2 & 47.1 & 13.3 & 13.4 & 26.1 & 26.3 & -3.8 & -3.9 & -7.4 & -7.6 & 51 \\
\hline${ }_{2} \mathrm{C}-\mathrm{CH}_{3}$ & 91.4 & 91.4 & 66.5 & 66.4 & 29.9 & 29.9 & 48.7 & 48.6 & 5.0 & 4.9 & 8.1 & 8.0 & 61.5 \\
\hline & 77.0 & 76.9 & 56.5 & 56.5 & 17.0 & 16.9 & 28.3 & 28.1 & -3.6 & -3.5 & -5.9 & -5.8 & 60 \\
\hline B & 9 & 77.1 & 5.3 & 65.2 & 8.9 & 9.1 & 13.1 & 13.3 & -2.8 & -2.8 & -4.0 & -4.1 & 68 \\
\hline $\mathrm{Cl}$ & 3.1 & 68.2 & 66.5 & 66.3 & -4.9 & -4.8 & -6.7 & -6.6 & -6.5 & -6.7 & -8.9 & -9.2 & 73 \\
\hline$-\mathrm{Br}$ & 98.1 & 98.3 & 78.4 & 78.4 & 20.6 & 20.8 & 26.6 & 26.9 & 0.9 & 0.9 & 1.1 & 1.2 & 77.5 \\
\hline & 93.3 & 93.1 & 77.2 & 77.3 & 17.3 & 17.1 & 22.7 & 22.5 & 1.2 & 1.3 & 1.6 & 1.7 & 76 \\
\hline Benzyl iodine/C-I & 92.3 & 92.3 & 57.0 & 56.9 & 29.1 & 29.1 & 46.0 & 46.1 & -6.2 & -6.3 & -9.8 & -9.9 & 63.2 \\
\hline
\end{tabular}

The determined reaction enthalpies values were quasiidentical to those obtained in the activation energies, computed using Transition States.

The HDE were computed from the TBE difference between geometrically optimized molecular structures and fragments resulted (geometrically optimized and unoptimized) in the process of chemical bonds breaking and those separations at more than $10 \AA$.

The accuracy of the HDE evaluation method was ver- ified by the results obtained, using quantum mechanical calculations inter-compared with experimental results identified in literature. As it can be observed in Table I, HDE values for hydrocarbons obtained by simulation, present similar values with those identified in literature [6]. For the method which uses geometrically unoptimized fragments, in comparison with experimental results we observe about $7 \%$ deviations just in the case of $\mathrm{C}-\mathrm{C}$ bonds and neighboring benzyl position bonds. 
In the case of chemical bonds in allyl or benzyl position (Table II), the best results were obtained if optimized fragments were used in calculations (which present identical TBE with those of transition states). As in hydrocarbons case the HDE for $\mathrm{C}-\mathrm{C}$ bonds presents $5-10 \%$ deviations from experimental results.

In the case of oxygenated, halogenated and those containing S compounds, (see Table III) HDE values, obtained using the method applied to allyl or benzyl compounds are similar with the experimental data.

The computed HDE and experimentally determined BE values [6] for oxygenated compounds.

TABLE III

\begin{tabular}{|c|c|c|c|c|c|c|c|c|c|c|c|c|c|}
\hline \multirow[t]{2}{*}{$\begin{array}{l}\text { Chemical compound } \\
\text { /Chemical bond }\end{array}$} & \multirow{2}{*}{\multicolumn{2}{|c|}{$\begin{array}{c}\text { HDE } \\
\text { unoptimized } \\
{[\mathrm{kcal} / \mathrm{mol}]}\end{array}$}} & \multirow{2}{*}{\multicolumn{2}{|c|}{$\begin{array}{c}\text { HDE } \\
\text { optimized } \\
{[\mathrm{kcal} / \mathrm{mol}]}\end{array}$}} & \multicolumn{4}{|c|}{$\begin{array}{c}\text { HDE } \\
\text { unoptimized deviation }\end{array}$} & \multicolumn{4}{|c|}{$\begin{array}{c}\text { HDE } \\
\text { optimized deviation }\end{array}$} & \multirow{3}{*}{$\begin{array}{r}\text { Exp. } \\
\text { BE } \\
{[6]}\end{array}$} \\
\hline & & & & & \multicolumn{2}{|c|}{$[\mathrm{kcal} / \mathrm{mol}]$} & \multicolumn{2}{|c|}{$g$} & \multicolumn{2}{|c|}{$[\mathrm{kcal} / \mathrm{mol}]$} & \multicolumn{2}{|c|}{$\%$} & \\
\hline Semi- & PM3 & AM1 & PM3 & AM1 & PM3 & AM1 & PM3 & 11 & PM3 & AM1 & PM3 & \pm & \\
\hline Acet & 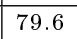 & 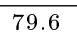 & 7 & 8.5 & 7.6 & 7.6 & 10.6 & & -7.3 & -3.5 & -10.1 & & 72 \\
\hline For & 79.8 & 79.9 & 74.3 & 76.5 & 3 & 3.9 & 5 & 5.1 & -1.7 & 0.5 & -2.2 & 0.7 & 76 \\
\hline & 77.6 & 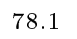 & 3.2 & & 2.6 & & & & -8.8 & -5.4 & -11.7 & -1.2 & 5 \\
\hline Ben & 1 & 82.3 & 75.3 & 73.9 & -6.9 & -5.7 & -7.8 & -6.5 & -12.7 & -14.1 & -14.4 & -16.0 & 88 \\
\hline & & .7 & 7.1 & 87.9 & 0.2 & 0.7 & 0.22 & 0.8 & -2.9 & -2.1 & -3.2 & -2.3 & 0 \\
\hline Acetic & 89.4 & 1 & 4.3 & 8 & -0.6 & 0.1 & -0.7 & 0 . & -5.7 & -5 & -6.3 & -5.6 & 90 \\
\hline Etha & 86.5 & 86.6 & 79.2 & 80.1 & -3.5 & -3.4 & -3.9 & -3.8 & -10.8 & -9.9 & -12.0 & -11.0 & 90 \\
\hline Met & 91.6 & 91.1 & $82 .^{\prime}$ & 83.4 & 0.6 & 0.1 & 0.66 & 0.1 & -8.3 & -7.6 & -9.1 & -8.4 & 91 \\
\hline & .5 & & .4 & 1 & & 2.3 & 33 & 2.2 & 4.4 & 3 & 4.2 & 2.9 & 104 \\
\hline ne & 46.5 & 46.4 & 46.5 & 45.9 & -4.5 & -4.6 & -8.8 & -9.0 & -4.5 & -5.1 & -8.8 & -10.0 & 51 \\
\hline & .7 & 30.1 & 29.5 & 29 & 2.7 & 1. & 9.31 & 3. & 0.5 & 0.8 & 1.7 & 2.8 & 29 \\
\hline Dimethy peroxide/O-O & 28.6 & 29.8 & 13.2 & 15 & -8.4 & -7.2 & -23 & -19.5 & -23.8 & -22 & $|-64.3|$ & -59.5 & 37 \\
\hline
\end{tabular}

The computed HDE and experimentally determined BE values [6] for halogenated compounds.

TABLE IV

\begin{tabular}{|c|c|c|c|c|c|c|c|c|c|c|c|c|c|}
\hline \multirow{3}{*}{$\begin{array}{l}\text { Chemical compound } \\
\text { /Chemical bond } \\
\text { Semiempirical method }\end{array}$} & \multirow{2}{*}{\multicolumn{2}{|c|}{$\begin{array}{c}\text { HDE } \\
\text { unoptimized } \\
{[\mathrm{kcal} / \mathrm{mol}]}\end{array}$}} & \multirow{2}{*}{\multicolumn{2}{|c|}{$\begin{array}{c}\text { HDE } \\
\text { optimized } \\
{[\mathrm{kcal} / \mathrm{mol}]}\end{array}$}} & \multicolumn{4}{|c|}{$\begin{array}{c}\text { HDE } \\
\text { unoptimized deviation }\end{array}$} & \multicolumn{4}{|c|}{$\begin{array}{c}\text { HDE } \\
\text { optimized deviation }\end{array}$} & \multirow{3}{*}{$\begin{array}{c}\text { Exp. } \\
\text { BE } \\
{[6]}\end{array}$} \\
\hline & & & & & \multicolumn{2}{|c|}{$[\mathrm{kcal} / \mathrm{mol}]$} & \multicolumn{2}{|c|}{ 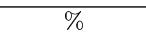 } & \multicolumn{2}{|c|}{$[\mathrm{kcal} / \mathrm{mol}]$} & \multicolumn{2}{|c|}{$\%$} & \\
\hline & PM3 & AM1 & PM3 & AM1 & PM3 & AM1 & PM3 & Alvit & PM3 & AM1 & PM3 & & \\
\hline & .7 & & & .3 & .3 & 1.8 & 3.2 & & & & 11.6 & & 03 \\
\hline & 13.8 & 112.5 & 10.5 & 110.2 & -7.2 & -8.5 & -6.0 & -7.0 & -10.5 & -10.8 & -8.7 & -8.9 & 121 \\
\hline & 4.1 & 175 & 3.9 & 4.2 & $0_{1}$ & 30 & 6.3 & 20.7 & -31.1 & -30.8 & -21.4 & -21.2 & 45 \\
\hline $\mathrm{CH}_{3}$ & 108.3 & 109.1 & 100.7 & 100.2 & 0.3 & 1.1 & 0.3 & 1.0 & -7.3 & -7.8 & -6.8 & -7.2 & 108 \\
\hline reo & 108.3 & 110 & 93.3 & 93.9 & -3.7 & -2 & -3.3 & -1.8 & -18.7 & -18.1 & -16.7 & -16.2 & 112 \\
\hline & 93.8 & 9 & 78.6 & 69.3 & -3.2 & -4 & -3.3 & -4.1 & -18.4 & -27.7 & -19.0 & -28 & 97 \\
\hline 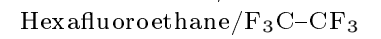 & 66.3 & 66.7 & 60.3 & 59.2 & -2.7 & -2.3 & -3.9 & -3.3 & -8.7 & -9.8 & -12.6 & -14.2 & 69 \\
\hline & 91.5 & & 1.4 & & 1.5 & 2 & 1 & 2.2 & -8.6 & -10.9 & -9.6 & -12.1 & 90 \\
\hline & 76 & .1 & 9.2 & 2 & -7 & -7.9 & -8.4 & -9.5 & -13.8 & -17.8 & -16.6 & -21.4 & 83 \\
\hline $\mathrm{CH}_{3} \mathrm{I}$ & 100.6 & 102.3 & 91.2 & & 1. & 3.3 & 1.6 & 3.3 & -7.8 & -7.2 & -7.9 & -7.3 & 99 \\
\hline & 52.2 & 5 & 9.8 & 44.6 & -0.8 & -1 & -1.5 & -1.9 & -3.2 & -8.4 & -6.0 & -15.8 & 53 \\
\hline & .4 & 4 & 44.2 & & -4.6 & -4 & -9.0 & -7.8 & -6.8 & -13.2 & -13.3 & -25.9 & 51 \\
\hline Iodobenzene/Ph-I & 53.5 & 54.7 & 51.9 & 52.3 & -3.5 & -2.3 & -6.1 & -4.0 & -5.1 & -4.7 & -8.9 & -8.2 & 57 \\
\hline
\end{tabular}

The values obtained for halogenated compounds present significantly fewer deviations especially in the case of some fluoride compounds (benzene-hexafluoride, methane trifluoride and trichloride compounds Table IV).

The HDE evaluation in compounds containing $\mathrm{N}$ and $\mathrm{S}$ (Table V) does not lead to satisfying results, the majority of obtained values having more than $7 \%$ deviation in comparison with experimental data.

\section{Conclusions}

A model for HDE determination by homolytic fragmentation processes simulation was proposed. The results obtained suggest two evaluation methods of HDE, depending on the neighboring chemical bond analyzed.

(i) In the neighboring position of aromatic groups or unsaturated bonds HDE can be determined as the difference between the TBE value of resulted fragments by chemical bond breaking, energetically minimized, and the initial molecular structure respectively.

(ii) In the other cases, HDE can be determined from the difference between TBE of geometrically optimized molecular structures and radical fragments energetically unminimized.

In homolytic fragmentation processes evaluation TBE was used because the determination of formation enthalpies is realized with low grade accuracy.

Transition states analysis has produced similar results with those obtained in the case of energetically minimized fragments use. Because that calculation procedure is much more difficult in the case of using Transition State-HYPERCHEM, the method is recommended only for those cases where doubts exist concerning energetic values obtained.

From all analyzed semi-empirical methods, PM3 and AM1 produced the best results, value differentiation be- 
The computed HDE and experimentally determined BE values [6] for nitrogen and sulfur compounds.

TABLE V

\begin{tabular}{|c|c|c|c|c|c|c|c|c|c|c|c|c|c|}
\hline \multirow{3}{*}{$\begin{array}{l}\text { Chemical compound } \\
\text { /Chemical bond } \\
\text { Semiempirical method }\end{array}$} & \multirow{2}{*}{\multicolumn{2}{|c|}{\begin{tabular}{|c|} 
HDE \\
unoptimized \\
{$[\mathrm{kcal} / \mathrm{mol}]$} \\
\end{tabular}}} & \multirow{2}{*}{\multicolumn{2}{|c|}{$\begin{array}{c}\text { HDE } \\
\text { optimized } \\
{[\mathrm{kcal} / \mathrm{mol}]}\end{array}$}} & \multicolumn{4}{|c|}{$\begin{array}{c}\text { HDE } \\
\text { unoptimized deviation }\end{array}$} & \multicolumn{4}{|c|}{$\begin{array}{c}\text { HDE } \\
\text { optimized deviation }\end{array}$} & \multirow{3}{*}{$\begin{array}{c}\text { Exp. } \\
\text { BE } \\
{[6]} \\
\end{array}$} \\
\hline & & & & & \multicolumn{2}{|c|}{$[\mathrm{kcal} / \mathrm{mol}]$} & \multicolumn{2}{|c|}{$\%$} & \multicolumn{2}{|c|}{$[\mathrm{kcal} / \mathrm{mol}]$} & \multicolumn{2}{|c|}{$\%$} & \\
\hline & PM3 & AM1 & PM3 & AM1 & PM3 & AM1 & PM3 & AM1 & PM3 & AM1 & PM3 & AM1 & \\
\hline Hydrazine/ $\mathrm{H}_{2} \mathrm{~N}-\mathrm{NH}_{2}$ & 50.3 & 52.1 & 49.8 & 50.2 & -9.7 & -7.9 & -16.2 & -13.1 & -10.3 & -9.8 & -17.1 & -16.3 & 60 \\
\hline $\mathrm{Am}$ & .8 & 94.1 & 90.4 & 91.1 & -10.2 & -7.9 & -10.0 & -7.7 & -11.6 & -10.9 & -11.4 & -10.7 & 102 \\
\hline Methyl & 81.0 & 83.4 & 70.4 & 70.1 & -10.0 & -7.6 & -11.0 & -8.3 & -20.6 & -20.9 & -22.7 & -22.9 & 91 \\
\hline Nitro $\mathrm{m}$ & 60.3 & 61.0 & 42.1 & 42.6 & 1.3 & 2.0 & 2.1 & 3.4 & -16.9 & -16.4 & -28.7 & -27.9 & 59 \\
\hline Methyl $n$ & 31.4 & 34.2 & 20.1 & 20.8 & -8.1 & -5.3 & -20.5 & -13.4 & -19.4 & -18.8 & -49.1 & -47.5 & 40 \\
\hline Acetc & 123.3 & 120.3 & 128.0 & 128.4 & 20.3 & 17.3 & 19.7 & 16.8 & 25.0 & 25.4 & 24.3 & 24.7 & 103 \\
\hline Met & 81.9 & 81.9 & 71.1 & 71.2 & 7.7 & 7.6 & 10.4 & 10.3 & -3.1 & -3.0 & -4.2 & -4.1 & 74 \\
\hline Eth & 73.4 & 74.5 & 60.8 & 60.7 & 0.0 & 1.1 & 0.1 & 1.5 & -12.6 & -12.8 & -17.1 & -17.4 & 73 \\
\hline $1 \mathrm{Pro}$ & 74.7 & 74.8 & 62.0 & 61.5 & 2.6 & 2.7 & 3.6 & 3.8 & -10.1 & -10.6 & -14.0 & -14.7 & 72 \\
\hline $2 \mathrm{Pr}$ & 67.8 & 67.5 & 52.9 & 53.1 & -2.8 & -3.1 & -4.0 & -4.4 & -17.7 & -17.5 & -25.1 & -24.8 & 71 \\
\hline Tert & 63.3 & & 46.1 & 46.7 & -6.1 & -6.4 & -8.7 & -9.2 & -23.3 & -22.7 & -33.5 & -32.7 & 69 \\
\hline Dimet & 77.1 & 77.2 & 66.0 & 66.3 & 3.9 & 4.0 & 5.4 & 5.5 & -7.2 & -6.9 & -9.8 & -9.4 & 73 \\
\hline 1 & 69.9 & & 56.8 & 56.4 & -1.9 & -1.7 & -2.6 & -2.4 & -15.1 & -15.4 & -21.0 & -21.4 & 72 \\
\hline Ethylmethy & 77.5 & 77.2 & 66.3 & 66.5 & 6.9 & 6.6 & 9.8 & 9.4 & -4.3 & -4.1 & -6.1 & -5.8 & 71 \\
\hline Diethyl thioether/C-S & 71.1 & 72.0 & 57.7 & 57.7 & 0.5 & 1.4 & 0.7 & 1.9 & -12.9 & -12.9 & -18.2 & -18.3 & 71 \\
\hline
\end{tabular}

ing minimal. PM3 method allows to reach the energy minimum much faster, while AM1 method presents setting incompatibilities in some cases [5]. For this reason, PM3 method is only recommended for use in exceptional cases for the compounds which contain allyl or benzyl groups, $\mathrm{N}$ or halogen atoms.

Computed values for $\mathrm{C}-\mathrm{H}, \mathrm{O}-\mathrm{H}$ bonds present minimum deviation (less than $5 \%$ ) in comparison with experimental values, while $\mathrm{HDE}$ values for $\mathrm{C}-\mathrm{C}$ bonds were obtained with a $5-7 \%$ deviation. In hydrocarbons case, with the exception of allyl or benzyl the deviation is exclusively positive.

Based on the obtained results we may conclude that the proposed method presents satisfactory accuracy, except for compounds containing nitrogen, sulfur and halogens.

\section{Acknowledgments}

This research is supported by the Project PNCDI II 177/2012 and Core Project No 09370206 from the Ex- ecutive Unit for Financing Education Higher Research Development and Innovation of Romania.

\section{References}

[1] G. Choppin, J.O. Liljenzin, J. Rydberg, C. Ekberg, Radiochemistry and Nuclear Chemistry (Fourth Edition), Academic Press, 2013, p. 209.

[2] C. Postolache, L. Matei, Radiat. Phys. Chem. 76, 1267 (2007).

[3] J.J.P. Stewart, J. Comput. Chem. 10, 209 (1989).

[4] M.J.S Dewar, E.G. Zoebisch, E. Healy, J.J.P. Stewart, J. Am.Chem. Soc. 107, 3902 (1985).

[5] C. Cojocaru, A. Rotaru, V. Harabagiu, L. Sacarescu, Journal of Molecular Structure 1034, 162 (2013).

[6] T.L. Cottrell, Numerical Values of Bond Dissociation. Energies in The Strengths of Chemical Bonds, edited by Butterworths Scientific Publication, London, UK, 1958. 